\title{
Diálogo de saberes en torno a la presencia de contenidos con perspectiva de género en la Universidad Nacional de San Juan y la Universidad Nacional de Córdoba
}

A dialogue of knowledge around the inclusion of topics with a gender perspective at the Universidad Nacional de San Juan and the Universidad Nacional de Córdoba

ISSN 2071-8748

E-ISSN 2218-3345

(c) (9) (2)

BY NC SA

DOI: https://doi.org/10.5377/entorno.v0i69.9560

URI: http://hdl.handle.net/11298/1154

\section{Resumen}

El objetivo de este trabajo es pensar el modo en el que se relaciona la perspectiva de género y feminista con las prácticas pedagógicas en la educación superior en la Universidad Nacional de San Juan y la Universidad Nacional de Córdoba. Ambas investigaciones exploratorias han formado parte de una misma línea de trabajo, y proponemos unir las indagaciones para encontrar puntos de contacto entre ambas universidades. Los resultados a los que hemos
Victoria Galoviche Universidad Nacional de San Juan-CONICET ORCID: 0000-0001-89458437

vicogalo@hotmail.com

Paola Bonavitta Universidad Nacional de Córdoba-CONICET ORCID: 0000-0003-4758-4202 paola.bonavitta@gmail.com

Andrea Analía Benavídez Universidad Nacional de San Juan ORCID: 0000-0002-8990-9645 andreabenavidez@unsi-cuim.edu.ar

Recibido: 19 de febrero 2020

Aceptado: 27 de junio de 2020

\section{Abstract}

The objective of this investigation is to think about the way in which the perspective on gender and feminism relates to the pedagogical practices in higher education at the Universidad Nacional de San Juan and the Universidad Nacional de Córdoba. Both exploratory investigations are part of the same line of work, therefore generating a proposal for joining both enquiries with the purpose of finding contact points between both universities. 
llegado muestran que la educación superior requiere la construcción de espacios de pensamiento y acciones que garanticen la inclusión de la perspectiva de género.

\section{Palabras clave}

Feminismo - Argentina; Formación profesional de mujeres - Argentina; Igualdad en la educación - Argentina; Perspectiva de género - Argentina.
The findings show that higher education requires the construction of spaces of thought and actions that guarantee the inclusion of the perspective on gender.

\section{Keywords}

Feminism - Argentina; Women profesional training Argentina; Equality in education - Argentina; Perspective on gender - Argentina.

\section{Introducción}

Este trabajo surge a instancia de proponer un diálogo entre investigaciones llevadas a cabo en la Universidad Nacional de Córdoba (UNC) y la Universidad Nacional de San Juan (UNSJ) donde se relevó la presencia de contenido con perspectiva de género en las asignaturas de las dos universidades. Los dos equipos estuvieron guiados por los mismos intereses, aunque con métodos de recogida de datos y análisis diferentes, de los que se da cuenta en el apartado metodológico. A pesar de las diferencias entre ambas instituciones, los puntos de encuentro resultan motivadores para establecer posibles continuidades sobre los temas que nos ocupan. En el desarrollo del trabajo, damos cuenta de cada uno de los abordajes realizados tanto en la UNSJ como en la UNC para centrarnos en la cooperación y no en la comparación de los datos obtenidos. Los puntos de encuentro entre ambas investigaciones están dados en la serie de preguntas que pueden hacerse; no ya en el aspecto metodológico que ha sido publicado de manera oportuna por cada uno de los equipos, sino en los aspectos comunes que tienen los trabajos (Benavídez et al., 2018; Bonavitta et al., 2018). También, nos planteamos desobedecer el imperativo metodológico que impone la comparación como estrategia privilegiada cuando se trata de vincular dos investigaciones con objetivos semejantes.

Los movimientos feministas han impulsado (a través de las olas y reivindicaciones) una profunda revisión en el modo en que se produce conocimiento, además de abrir campos teóricos nuevos que estimulan, en el contexto de la educación superior, la inclusión de las mujeres y las personas de la diversidad sexual como sujetos excluidos o marginados en los procesos histórico-políticos (Lamas, 1986, 2013; Bartra, 2018; Pérez Sedeño, 2001; Morgade, 2018). Este abordaje exploratorio en ambas universidades se centró en indagar en el modo en el que se relaciona la perspectiva de género y feminista con las prácticas pedagógicas en la educación superior. También es necesario profundizar sobre el camino que han seguido los/las docentes que abordan contenidos con perspectiva de género en las aulas a tomar esos posicionamientos; e indagar los métodos y medios didácticos elegidos para concretar este abordaje en cada clase.

El concepto de género, en el sentido amplio con el que nos hemos aproximado a los/las profesores, favoreció que emerjan diversas referencias teóricas. Se partió de considerar al género tanto en la historicidad del concepto como en las discusiones e invalidaciones de su ambiguo uso (Lamas, 1986, 2013; Bartra, 2018; Blanco, 2018). Asimismo, hemos podido poner en contacto las posibles situaciones comunes que se dan en ambas universidades, las referencias a marcos teóricos que tienen puntos de contacto y las prácticas políticas que favorecen esos posicionamientos en relación con el contexto situado. En ese sentido es que nos interesamos por abordar este trabajo desde una aventura de encuentros posibles. La realización de cada una de las investigaciones está marcada por modos sabidos y legitimados en el ámbito académico, pero nos proponemos, con la escritura de este trabajo, avanzar sobre sendas menos conocidas para buscar otros modos posibles, que no sean solo comparativos. Se trata de producir saberes desde una episteme que comprenda a la cooperación como modo posible de establecer diálogos proteicos. 


\section{Metodología}

Este artículo tiene su origen en las reflexiones surgidas frente a algunos datos relevantes contenidos en dos estudios de tipo exploratorios en la UNC y la UNSJ, que proponen relevar la presencia de contenidos con perspectiva de género en las asignaturas que se dictan en estas instituciones.

Respecto a la investigación citada de la UNSJ, el camino metodológico seguido fue principalmente cualitativo, por medio de entrevistas semiestructuradas a docentes de esta universidad que imparten contenidos con perspectiva de género en sus asignaturas en carreras de grado, acompañado por unas encuestas tomadas al comienzo del estudio, -a estudiantes y directores de carrera, para detectar las materias donde se desarrollaban estos temas.

En la UNC —como señalaremos más adelante-, se utilizó la metodología cuantitativa para acceder a los datos y se entrecruzó con el análisis cualitativo de contenido. Esto nos permitió pensar en los planes de estudio y sus recortes centrales. Indagar acerca de los planes de estudio oficiales de la Casa de Trejo (como se denomina a la UNC) nos facilita la comprensión acerca de cuáles son los contenidos válidos en la Universidad, qué es lo que se permite dictar en clases y qué perspectiva o perspectivas se fomentan. El enfoque que guía, tanto el relevamiento como el análisis de datos, se apoya en una perspectiva feminista, en relación con la práctica pedagógica en la educación superior.

\section{Notas sobre pedagogías feministas latinoamericanas}

Las fuentes de pedagogías feministas en Latinoamérica no son fáciles de rastrear. Como le acontece a todo lo que tiene que ver con los estudios de género, y a dichas pedagogías, el manto de invisibilidad amenaza con cubrir las nuevas prácticas que, en torno al conocimiento con perspectiva de género, emergen. Hay antecedentes no estrictamente feministas, como el de Paulo Freire: La naturaleza política de la educación. Cultura, poder y liberación, Pedagogía de la indignación. En un paradigma reproductivo de conocimientos mecanicista, la crítica no forma parte de los supuestos epistemológicos para pensar la educación. En cambio, el punto de partida para el pensamiento crítico que suponen los enfoques de pedagogías feministas es ya una posición ética, política y educativa que no puede ser soslayada. Podemos pensar que las prácticas pedagógicas emergentes se contraponen a las que sostienen el disciplinamiento a través del conocimiento, además de los papeles establecidos de manera inalterable mediante la jerarquía en el conocimiento y en el aula. Ante ese modo aludido, en el que las instituciones educativas normalizan tanto a sujetos como a conocimientos, es que emergen como propuesta los estudios de género y los enfoques feministas que cuestionan lo establecido, además de abrir las tramas de discusiones. Emerge también el cambio en la realidad social que tanto docentes como estudiantes proponen al espacio educativo universitario. En medio de esas luchas es que los estudios de género aparecen como modos de discutir la forma hegemónica y tradicional de plantear la relación enseñanza-aprendizaje en las universidades. Los movimientos en torno al género no son homogéneos, no Ilevan una línea de fácil identificación y no proceden de una sola fuente. Dentro de los estudios de género, el feminismo ha planteado una discusión tan profunda para la educación académica que las derivas dan consecuencias notables.

En la relación de ciencia y género, algunas de las urgencias han tenido que ver con cuantificar el ingreso y la permanencia de mujeres, y las sexualidades disidentes, a los espacios de formación, gestión y producción académica. Estos trabajos datan de la década del 60 al 70. En ese sentido, en Argentina Dora Barrancos (2002) ha sido pionera junto con María Luisa Femenías (2011); Zulma Palermo (2007); Gloria Bonder (1998); el grupo RAGCyT, integrado por Kochen, S., Franchi (2001), entre otras académicas, además de Diana Maffía (2007).

Otras de las urgencias han estado marcadas por la relación tensa entre universidad y sociedad, tanto que han abierto la agenda de la política académica puesta en discusión desde la Reforma Universitaria (1918). En ese sentido, el tipo de problemas teóricos provenientes de situaciones sociales ha sido un campo de lucha en que la comunidad educativa universitaria se ha hecho eco de diferentes formas. La situación de mujeres pobres o con discriminaciones diversas, debido a su raza o clase, ha sido reconocida en trabajos notables por autoras como Claudia Korol (2017), Francesca Gargallo (2008), Mara Viveros Vigoya (2009) y Gloria Anzaldúa (1989), entre otras.

También estamos pensando en las acciones colectivas como "Ni una menos", que están, ya, formando parte de la agenda académica durante esta década. De este modo, la universidad pudo ampliar la puerta de ingreso de lo social 
a las aulas desde los años 80 y 90 . Junto con el acceso paulatino de mujeres a cargos de profesoras titulares, a espacios de investigación jerarquizados y de gestión, se abrió la posibilidad para que la adscripción al género fuera posible en el tratamiento de teorías y temas considerados por los movimientos feministas. De la misma manera, el acceso a estudios de posgrado permitió que los/las profesores tuvieran, a través del enfoque de género, acceso a visibilizar la bibliografía escrita por feministas dentro de la academia. Algunas de las autoras que han estudiado esta presencia de los reclamos sociales propios del feminismo en el ámbito de las aulas, entre otras, son Graciela Morgade $(2011,1997)$; Norma Blazquez Graf (2008) y Patricia Castañeda (2016).

Otras perspectivas que en la academia aportan a la discusión de los feminismos latinoamericanos son la propuesta por Espinoza, Yuderkys (2013), Catherine Walsh (2009), Breny Mendoza (2014) y Francesca Gargallo (2008); y las procedentes de las chilenas Amanda Labraca (1947) y Julieta Kirkwood (1987); y de la boliviana Antonia Irayigra (2012) y Ana Buquet (2011), entre otras referencias que pueden ser mencionadas.

Estado actual de las prácticas áulicas e inclusión de temáticas de género en la Universidad Nacional de San Juan

Respecto al análisis para la UNSJ, se presenta aquí una serie de datos interesantes respecto al género y su vinculación con las asignaturas en las Carreras de Grado UNSJ. Se parte de un estudio realizado por el equipo GAGES ${ }^{1}$ sobre la presencia de contenidos con perspectiva de género en las diferentes asignaturas en el período 2017-2018, indagando en el conjunto de temáticas abordadas por los/las docentes $\mathrm{y}$ las formas de desarrollarlas en el aula.

En la UNSJ, podemos advertir que en la actualidad esta es una de las cincuenta y seis (56) universidades nacionales públicas que hay en Argentina, y se encuentra emplazada en la provincia de San Juan, Argentina; ubicada en la zona cordillerana al oeste del país. San Juan posee un total de 755.994 habitantes. Por su parte, la UNSJ posee una matrícula total de 22.057 alumnos/as.
Respecto a su estructura actual, la UNSJ cuenta con cinco Facultades: Ingeniería; Arquitectura, Urbanismo y Diseño; Ciencias Exactas, Físicas y Naturales; Ciencias Sociales; y Filosofía, Humanidades y Artes. Cada una de ellas tiene en su interior departamentos académicos; a la vez que institutos, centros, gabinetes y museos de investigación y extensión. Asimismo, la UNSJ posee tres escuelas de nivel medio. El gobierno de la UNSJ está integrado por autoridades ejecutivas, entre las que se incluyen jerárquicamente, Rector/a y Vicerrector/a de la Universidad, decanos/as y vicedecanos/as de Facultades, directores/as y vicedirectores/ as de departamentos, y directores/as y vicedirectores/ as de colegios de nivel medio. También está integrado por Ios siguientes órganos colegiados: Asamblea Universitaria, Consejo Superior, Consejos Directivos de Facultad, Consejos Departamentales y Consejos Asesores Escolares.

Desde la UNSJ, la problemática de la desigualdad de género ha sido una inquietud desde hace algunos años. Se evidencia en la propuesta del un Protocolo de actuación en la UNSJ ante situaciones de discriminación, violencias a la identidad de género y contra las mujeres, que fue aprobado en 2016 por la Ordenanza N. 019 del Consejo Superior de esta casa de estudios. Su aprobación fue sincrónica con la creación de la Oficina por la igualdad de género, contra las violencias y la discriminación, que depende de la Secretaría de Bienestar Universitario. Cabe resaltar que este protocolo es uno de los primeros en universidades del país. Asimismo, se puede advertir la presencia de variados equipos de investigación y recientes tesis de grado y posgrado que indagan sobre el género y sus condiciones desde diferentes miradas.

Ahora bien, el relevamiento de información propuesto por este estudio representa, para esta universidad, un insumo que permite problematizar y reflexionar sobre el lugar que tiene el género en la educación superior aquí. El total de asignaturas relevadas fue de 47 , que equivale a un $2 \%$, aproximadamente, en toda la Universidad. Entre los principales resultados, podemos advertir que en la UNSJ la Facultad de Filosofía, Humanidades y Artes (FFHA), con un total de 19 materias, es la unidad académica en la que se aborda la temática de género en mayor cantidad de

\footnotetext{
1 Estudio "Presencia de contenidos de género en carreras de grado: El caso de la UNSJ", realizado por Equipo GAGES Grupo de Articulación en Género y Educación Superior , perteneciente a la Universidad Nacional de San Juan en articulación con la Universidad de Alicante. Integrantes: Andrea Benavídez, Valeria Gili Diez, Victoria Galoviche, Paula García Mavrich, Mariana Guerra., Franco Barboza Pirán, Ivana Soler, Juan Mattar, Hernán Videla y Gabriela Bazán.
} 
materias. Esto puede estar relacionado con el hecho de que es la Facultad que concentra a las carreras de formación docente y profesorados que se dictan en la UNSJ, donde los/ las docentes de las asignaturas pedagógicas y humanísticas tratan el género como una temática recurrente.

Por otra parte, la Facultad de Ciencias Sociales (FACSO) tiene 16 asignaturas donde se abordan contenidos con perspectiva de género. Las Facultades de Ingeniería, y de Ciencias Exactas, Físicas y Naturales (FCEFyN), y la Escuela Universitaria de Ciencias de la Salud (EUCS) representan una menor cantidad de asignaturas $(5,3$ y 4 , respectivamente) donde se aborda la temática. Esto podría relacionarse con que el objeto de estudio disciplinar de las carreras que sirven estas Facultades se distancian de las áreas donde generalmente la temática de género se encuentra en debate.

Los/las docentes que fueron entrevistados/as (43) como puede observarse son de variadas carreras de grado, con perfiles y formaciones diferentes. Docentes con formación de grado y postgrado, tanto varones como mujeres, y de diferentes edades. Algunos/as acompañan la práctica del aula con militancia en grupos o movimientos de género.

En el desarrollo de las entrevistas, se preguntó de manera abierta sobre "contenidos relacionados con la temática de género" de un modo general, esperando la respuesta espontánea de los/las docentes, quienes comprenden en su mayoría que tratar temáticas de género en las aulas es hablar de los condicionantes en forma de desigualdades e inequidades que en el género se sustentan.

Con base en este relevamiento, se puede decir que el conjunto de contenidos conceptuales tratados en las diferentes asignaturas, según observamos en las planificaciones de las materias seleccionadas, como así también en el discurso de docentes entrevistados/as, emerge con dos modalidades específicas que se vinculan entre sí. Por una parte, aquellos que aparecen como currículos formales, explicitados en las planificaciones de las asignaturas junto con los contenidos procedimentales y actitudinales que conforman el proceso de enseñanza-aprendizaje. Por otra, aquellos que se manifiestan en el currículo oculto, entendiéndose por este el conjunto de contenidos, estrategias, discursos que se transmiten efectivamente en las aulas, pero que no son explicitados en el plan de estudios.
Los contenidos conceptuales son diversos y se encuentran atravesados tanto por el campo disciplinar específico como por preocupaciones teóricas comunes. Es el caso de docentes de la Licenciatura en Enfermería, quienes explicitan que trabajan "Temáticas dentro de lo que es género, el trato de los profesionales médicos con la enfermería, el trato entre profesionales. El rol laboral (...)", situación que se repite en la Licenciatura en Historia en donde abordan la " (...) historia de mujeres e historia del género", "Historia americana I, que es en realidad Historia colonial, género - patriarcado".

Entre las temáticas abordadas en las asignaturas, y que se desprenden de las entrevistas realizadas a los/las profesores, podemos enunciar las siguientes:

- Estereotipos vinculados con el género y División sexual del trabajo

- $\quad$ Sistema patriarcal machista

- Violencia de género y Abuso sexual

- Prostitución. Trata sexual y laboral

- Partidos políticos, Políticas públicas y género

- Organizaciones sociales y Movimientos de mujeres

- Lenguaje no sexista. Género y análisis discursivo

- Tratamiento mediático de temas de género

- Conceptualizaciones en torno al género

- Prácticas laborales signadas por el género. Mujer y trabajo. Brecha Salarial

- Identidad de género

- Brechas en el acceso a tecnologías de la información

- Apropiación del cuerpo/corporalidades

- Raza, clase y género

- Política sexual de la carne

- Mujer y campo científico

- Normativas provinciales y nacional en torno al género

El listado de dimensiones, que de manera precedente señalamos, son expresiones de sentido textuales del total de docentes entrevistados/as en la UNSJ. Es importante explicitar que en algunos casos encontramos un abordaje de contenidos con perspectiva de género de manera 
transversal. En alusión a ello, una docente manifestó que "para estas dos cátedras la perspectiva de género es transversal, porque es cómo miramos, cómo seleccionamos, cómo focalizamos y cómo escribimos". En este sentido, con transversalidad de género aludimos a las asignaturas que trabajan las temáticas de género no como un contenido aislado, sino como contenido que se deriva de una normativa interna de cada departamento en las Facultades analizadas, como plan de estudio. Ejemplo de este tipo de encuadre lo representa la licenciatura en Comunicación Social, de la Facultad de Ciencias Sociales, que a partir de 2015 modificó su organización curricular, añadiendo el enfoque de género y los derechos humanos a su carrera. Así lo expresa una docente: “(...) Se trabaja (...) bajo el marco de derechos humanos, y bajo este gran paradigma, está la perspectiva de género"; . "Se encuentra al nivel plan de estudios del departamento. Nos lleva a tener esa perspectiva de género".

También hallamos aquellas/os docentes que manifiestan otro tipo de transversalidad, la que surge de proponer temáticas de género en todas las unidades por abordar en la materia, es decir, el género visto como un contenido transversal relacionado desde el tema 1 hasta el final de la cátedra. Lo observamos en el siguiente comentario docente:

Los contenidos degénerosonabsolutamente transversales a cada una de las unidades. Por ejemplo, ahora estamos viendo la primera unidad; que es cuando hablamos del estudio de Ingeniería Industrial. Entonces trabajamos mucho el tema de las brechas que hay para que se pueda realizar la sociedad del conocimiento [...]. En la segunda unidad, es empresa. Trabajamos todo el tema de los desafíos que tiene la mujer para dirigir y liderar procesos de gestión de empresas. Después pasamos a otras...

Es posible pensar que estas expresiones ponen en evidencia posicionamientos epistemológicos y políticos que atraviesan los contenidos formales $u$ ocultos en el espacio curricular; fundamentalmente porque el lenguaje es una de las principales maneras que utilizamos para nombrar y darle forma a nuestro mundo. En muchos de estos casos, las formas de citar los contenidos poseen ya una carga teórica importante; se trata de categorías analíticas relevantes en los debates de género.

Los posicionamientos epistemológicos son el cimiento desde el cual los/las docentes entienden y objetivan los contenidos conceptuales formales u ocultos en las planificaciones de las asignaturas. En cuanto a estos, se observa que principalmente refieren a cuerpos teóricos que comprenden la colonialidad, pos(de)colonialidad, feminismos y la reconstrucción epistémica de la historia del género.

Estas perspectivas fueron encontradas mayoritariamente en las asignaturas de las Ciencias Sociales y Humanas, como, por ejemplo, la Licenciatura en Filosofía, Licenciatura en Comunicación y Licenciatura en Trabajo Social. Una docente expresaba claramente cómo se articula el posicionamiento epistemológico con los contenidos conceptuales abordados en la asignatura como sigue:

Bien. Me gustaría, un poco, hacer el encuadre epistemológico de la materia (...) Yo hago un marco teórico desde el giro decolonial. Entonces, para nosotros la filosofía de la liberación y la emancipación del pensamiento es fundante. Entonces, al trabajar esa colonialidad del poder, del saber y del ser, de suyo aflora esta perspectiva de género (...) Entonces, imagínese que, dentro de los ítems para desarrollar el programa, tenemos esa perspectiva de género.

En la mayoría de los casos, si bien la postura epistemológica o teórica no fue explicitada, podemos inferir que han sido construidos a partir de sus propias formaciones disciplinares además de las experiencias personales y trayectorias docentes. Cabe advertir que, en las respuestas de los/las entrevistados/ as, no se apreciaron diferencias significativas en cuanto a sus expresiones de sentido en relación con el propio sexo/género o nivel de formación. Lo que nos habla de que el género es un aspecto transversal en la práctica de estos docentes dispuestos a problematizarlos en aula. La preocupación por abordar las temáticas de género está presente, y se desarrollan variadas formas para acercarlas a los/las estudiantes según señalaron en los encuentros.

\section{Estrategias didácticas desarrolladas}

Las estrategias didácticas aplicadas en clase para desarrollar, contar y compartir los contenidos de género son variadas: lectura comprensiva, abordaje teórico de conceptos, producción de textos, prácticas grupales, escritura, exposición, técnicas para conocer saberes previos en los estudiantes (lluvia de ideas, preguntas disparadoras), análisis de información en medios de comunicación (local y nacional), análisis de contenidos audiovisuales (películas, videos, sitios de internet, 
publicidades, canciones, cortos), análisis de documentos oficiales (datos estadísticos, leyes, políticas públicas); presentación de experiencias personales en género (análisis de casos), debate dirigido sobre distintos tópicos, producción de contenidos en comunicación y obras musicales, charlas informativas en el aula (con la presencia de especialistas en la temática), escucha atenta, formas dinámicas en que se organiza el espacio-aula: disposición del aula en círculo.

En cada caso, las clases se van formando en un proceso continuo, donde se va definiendo el contenido pedagógico; este incluye "las formas de representar y formular la asignatura que la hacen comprensible para los demás" (Putnam y Borko, 2000). Este proceso comprende, además: a) la concepción global de la docencia de una asignatura, b) el conocimiento de las estrategias y representaciones sobre la instrucción, c) conocimiento de las interpretaciones, el pensamiento y el aprendizaje de los/las estudiantes de una asignatura, y d) el conocimiento del currículo y los materiales curriculares.

Puede decirse que, en la definición de las estrategias didácticas utilizadas por los/las docentes analizados/as, se recupera el entramado de estos procesos, sumando a ello la propia práctica educativa más amplia, que incluye no solo lo que pasa en el aula sino fuera de ella -antes y después de las clases-.

En los casos analizados, más allá de que las técnicas utilizadas son generalmente habituales en la educación moderna, se reconoce la idea de generar un tipo de pensamiento especial. Un conocimiento más bien ligado a la reflexión crítica y a la praxis contextualizada. Citamos aquí algunas de estas expresiones de sentido de los/las docentes:

Tratar de que emerja de entre los intercambios de ellos [estudiantes] un posicionamiento que venga hacer una construcción colectiva (Docente Facultad de Filosofía, Humanidades y Arte-UNSJ).

Sobre todo, tratar de que ellos [estudiantes] piensen por sí mismos, que traten de identificar estas desigualdades, estas diferencias en su propia vida cotidiana (Docente Escuela de la Salud-UNSJ).

Para mí es muy importante partir de la propia experiencia, de la singularidad de los alumnos porque no me interesa adoctrinar; y me interesa que ellos puedan repensarse desde su propia singularidad, desde su propia situación social y personal (Docente Facultad Ciencias Sociales-UNSJ).
Se comprende pues que el género "no se enseña", sino más bien se comparte, se narra y se vivencia. Así, Bárcena Orbe, Larrosa Bondía y Mèlich Sangrá (2006), cuando relacionan la educación con lo experiencial, se refieren a lo siguiente:

La experiencia es un viaje. Y, por eso, pensada como experiencia, la educación es una salida hacia un afuera donde no todo puede planificarse ni programarse. Se trata de un viaje en el que se hace una experiencia, la de una confrontación con lo extraño; la que consiste, también, en escapar de las identidades fijas e inmutables, desligarse, en fin, de los lazos que fueron impuestos. El viaje, pues, como experiencia, como salida que nos confronta con lo extraño y como posibilidad de un nuevo comienzo (237)

Respecto a las relaciones de género, las clases en las aulas de educación superior que revisamos se tornan de un modo diferente y distintivo: son momentos donde se despliegan estrategias "entrañables". Así como indica Marcela Lagarde (1999) cuando analiza los liderazgos de mujeres, y entiende que son "liderazgos entrañables", así podemos pensar a estas estrategias didácticas en el aula respecto a las temáticas de género. "Entrañables", pues se definen "con las entrañas, con el corazón, con lo que somos y lo que queremos ser. Porque somos esenciales para la vida, $y$, sobre todo, porque somos portadoras de alternativas para hacer viables a la sociedad y al mundo" (4).

Más allá del género del grupo humano con el que se trabaje en el aula, estas son entrañables también porque se van delineando, en muchos casos, en función de las propias vivencias de desigualdad relacionadas con el género vividas por docentes y estudiantes. No puede desconocerse que, en cada clase descripta tanto para docentes como estudiantes, juega un papel fundamental el "contar experiencias" en relación con temáticas de género.

\section{La perspectiva feminista en la Universidad Nacional de Córdoba}

En la UNC, en el año 2007, se creó el Programa de Género de la UNC; y uno de los objetivos consiste en: "aportar a la transformación de las condiciones de desigualdad e inequidad, existentes en el campo de los derechos humanos de mujeres, varones y otras identidades sexo-genéricas, tanto en el ámbito universitario como por fuera de él". 
En 2014, se institucionalizó el Área de Feminismos, Género y Sexualidades (FemGeS) en el Centro de Investigaciones de la Facultad de Filosofía y Humanidades como resultado de las diversas acciones llevadas a cabo por el Programa Interdisciplinario de Estudios de Mujer y Género . El FemGeS fomenta investigaciones, congresos, conversatorios, encuentros y cursos de grado y posgrado feministas en el marco de la Universidad, así como fomenta acciones positivas con perspectiva de género.

Además, en la UNC se aprobó la Ordenanza 9/11, que reconoce la identidad de género adoptada y autopercibida de cualquier persona a su solo requerimiento, aun cuando esta no coincida con su nombre y sexo registrales. Esto implica que los y las estudiantes de la UNC pueden tener consignado en su libreta $\mathrm{y} / \mathrm{o}$ credencial estudiantil el nombre elegido. En el año 2015, se implementó el Plan de acciones para erradicar las violencias de género en la UNC por resolución del Consejo Superior (1011/2015) a los efectos de "promover en la comunidad universitaria un ambiente libre de violencias de género y discriminación de cualquier tipo, por razones de género y/o identidad sexual". Asimismo, dentro de la UNC se está actualmente trabajando en la incorporación del cupo laboral trans, para que puedan acceder a trabajos rentados dentro de la institución y se permita la solvencia económica de las personas trans.

Por otra parte, algunas carreras, como Psicología, Trabajo Social, Sociología y Ciencia Política, han inaugurado en los últimos dos años los baños mixtos, donde el género no es un determinante al momento de ingresar. Este tipo de acciones positivas fomentan el respeto por las diversidades y disputan espacios de poder en el marco institucional. No obstante, los planes de estudio no parecen adaptarse a estas realidades y políticas que va adoptando la institución, al menos en lo que respecta al currículo visible y explícita en los planes de estudio oficiales.

En este marco, en la UNC realizamos ${ }^{2}$ un estudio, prioritariamente cuantitativo, que pretendió reconocer la presencia o no de asignaturas que efectivamente incorporen la perspectiva de género e incluyan temáticas referentes a los estudios de género en los planes de estudio. En primer lugar, recolectamos información estadística básica (anuarios de la UNC, INDEC, observatorios de violencia de género). En esas fuentes, recuperamos datos estadísticos acerca de los planes de estudios de la UNC, tanto en los estudios de grado como en los de posgrado. El diseño metodológico se realizó a partir de un cuestionario básico donde se analizó, por carrera y programas de pregrado, la oferta académica de la UNC. Fue implementado en una muestra de 214 programas de pregrado y posgrado como población total de la oferta académica de la Universidad.

Los cuestionarios tuvieron como propósito principal identificar las materias sobre género que pudieran existir en cada uno de los planes de estudio de las 15 Facultades en sus diferentes grados académicos: licenciatura, maestría, doctorado y especializaciones, respectivamente, así como las horas destinadas a dichas materias. Posteriormente, obtuvimos una base de datos que nos permitió sistematizar la información encontrada en las 15 Facultades existentes. A partir de allí, se elaboró un cuestionario estadístico y se extrajeron resultados clave para poder generar indicadores precisos.

A partir del análisis de datos, pudimos observar que solo cinco de las 15 Facultades han autorizado en su currículo explícito las materias sobre género y/o materias feministas. Esto es, que referenciaron explícitamente en sus programas de estudios explícitos la existencia de materias con perspectiva de género. Estas Facultades son: Ciencias Sociales, Ciencias Médicas, Derecho, Ciencias de la Comunicación y Filosofía y Humanidades. De todas maneras, debemos tener en cuenta que la Facultad de Ciencias Sociales cuenta con el Doctorado en Estudios de Género, que tiene 12 de las 14 materias con perspectiva de género de toda la Facultad. Las dos materias restantes se reparten en maestrías y licenciaturas.

Otro dato que hemos podido relevar es que el $40,63 \%$ de las materias que tienen perspectiva de género se dictan en las licenciaturas de la UNC; y el $37,5 \%$, en doctorados. Esto nos habla del interés de la Universidad en dar formación de género desde los estudios de grado. Si bien no se alcanza la transversalización, se apunta a que tengan fuerte presencia al menos en las carreras que optaron por incluir la perspectiva feminista en los planes de estudio. En el caso

2 Un equipo de investigación integrado por Paola Bonavitta, Jeli Camacho Becerra, Jimena de Garay Hernández, Cecilia Johnson, Gabriela Bard Wigdor, María Eugenia Gastiazoro, Luisa Fernanda Muñoz Rodríguez, Gabriela Artazo, Sofía Menoyo y Laura Sarmiento. La investigación se llevó a cabo durante 2016 y 2017. 
de los doctorados, debemos recordar que la UNC cuenta con el Doctorado de Estudios de Género, que concentra prácticamente la totalidad de las asignaturas mencionadas. Pensamos que, el que la oferta académica incluya el estudio de posgrado mencionado, da muestras de que se intenta abrir las puertas de la Casa de Trejo ${ }^{3}$ al feminismo.

En relación con la obligatoriedad o no de las materias, podemos destacar que la mayoría de las asignaturas que existen sobre género son obligatorias (78\%). Por tanto, existe un interés institucional en que los estudios de género sean conocimientos que debe adquirir todo el estudiantado. La excepción es Ciencias de la Comunicación, donde la mayoría de las materias con perspectiva de género son opcionales. Más allá de las carreras que sí cuentan con la perspectiva feminista, no debemos dejar de notar que solo un tercio de toda la UNC incluye en su currículo oficial materias que tengan perspectiva de género. Asimismo, el género no es transversal a todas las carreras ni es contenido obligatorio. Esto nos habla de las decisiones que la institución toma en relación con las problemáticas de las que da cuenta el movimiento feminista. También pueden considerarse las decisiones de política académica que no son tomadas con respecto a aquello que la sociedad está reclamando en movimientos sociales y acciones colectivas (NiUnaMenos, Vigilias por la Legalización del Aborto, etc.).

La UNC continúa sosteniendo un perfil sexista y androcéntrico, pues las mujeres y los estudios feministas no tienen una participación real y formal en el plano académico; no forman parte del currículo explícito y tampoco son incluidos en las bibliografías utilizadas. Más allá de algunas medidas puntuales que intentan fomentar la inclusión y que mencionamos más arriba, tampoco participan las personas LGTBQI (lesbianas, gais, transexuales (o transgénero), bisexuales, queer e intersexuales) del currículo universitario ni de la enseñanza. No existen materias dedicadas a las diversidades, y en carreras como Psicología se sigue estudiando a las diversidades como trastornos. Entonces, ¿podemos pensar realmente en una inclusión y en saberes equitativamente contribuidos?

Amelia Valcárcel (2008) aseguró que vivimos en el "espejismo de la igualdad", es decir, que la supuesta libertad e igualdad de las mujeres está limitada por abajo y por arriba; también por el medio. "Dentro del espejismo de la igualdad, es violencia apartar a las mujeres de los resortes de poder, de autoridad y respeto" (Valcárcel, 2008).

Para Mara Viveros Vigoya (2009), el racismo y el sexismo de los enfoques científicos dominantes naturalizan la diferencia y la desigualdad social, argumentando que las relaciones sociales son biológicas y basadas en las diferencias fenotípicas (como el color de piel). A su vez, ambas autoras asocian el dato corporal con la realidad social y caracterizan a los grupos que no son dominantes como predispuestos a la sumisión. En gran parte, es lo que viene sucediendo con la educación superior y en el ámbito académico de la UNC. Puesto que, ante la ausencia de políticas académicas con perspectivas de género que contemplen los enfoques feministas, se generan saberes pensados para varones cis, blancos, burgueses, heterosexuales, dejando fuera la amplia diversidad de modos de ser, estar y percibir el mundo.

Cuando la educación superior no se piensa, se crea, se ejecuta y se materializa desde una perspectiva feminista, las inclusiones no son posibles. En esos casos, las instituciones académicas siguen reproduciendo reglas propias del patriarcado que no contemplan la equidad.

Como los planes de estudio no incluyen al feminismo, a las mujeres y disidencias, pensamos que no es el interés de la academia focalizarse allí. Y, por ende, sigue siendo espacio de reproducción del saber hegemónico masculinizado. La Organización Internacional del Trabajo [OIT] (2016) sostiene: "En la práctica, la educación sigue siendo una institución social que refleja y reproduce las desventajas socioeconómicas y culturales prevalecientes en el resto de la sociedad" (3). Dado que esta sociedad es patriarcal, misógina y sexista, la educación superior no hace más que reproducir estos valores, prejuicios y estereotipos.

Tensiones entre el horizonte propuesto por las pedagogías feministas en los casos de la UNC y UNSJ

Hay diferentes miradas para analizar las formas pedagógicas respecto al abordaje de los contenidos con perspectiva

3 Así se conoce coloquialmente a la UNC, pues la calle Obispo Trejo fue el lugar adonde funcionó la primera sede de la institución, que, durante dos siglos, fue la única universidad del país. 
género, pero no podemos hablar de una sola pedagogía de género o perspectiva feminista, sino más bien de un proceso teórico en construcción. En otras palabras, se comprende que los distintos marcos teóricos resultado de amplios análisis y debates dan origen a formulaciones de acciones y estrategias para el logro de una equidad e igualdad de género en los espacios educativos.

Por lo expuesto en cada contexto de las universidades aquí investigadas, se pueden observar algunos puntos de acuerdo que definen el trabajo en las aulas, que responden al desarrollo de prácticas educativas que al menos en una etapa inicial y aun signadas por una cultura educativa patriarcal resultan respuestas disruptivas, que propician la reflexión y sensibilización sobre las desigualdades de género.

En el contexto de la UNSJ, la posición de los/las docentes en el aula da cuenta de variadas formas de transmitir y hacer posible los debates en torno al género. No siempre los/las docentes adscriben a una determinada teoría pedagógica, aunque en muchos casos las prácticas áulicas, así como las temáticas elegidas, son en sí mismas contra-hegemónicas con respecto a las pedagogías usadas tradicionalmente en la academia. Esto es, por su intento en la interpelación y la mirada crítica respecto a las desigualdades presentes.

Dos de las preguntas que nos motivan: ¿por qué los/ las docentes no apelan a teorías pedagógicas con perspectiva de género?, y, en caso de hacerlo, ¿en qué momento son explicitadas en las aulas? Encontramos que en general esa explicitación no sucede. No obstante, las formas de enseñanza y aprendizaje suponen abordajes teóricos vinculados con teorías de género, feministas, masculinidades, diversidad sexual y demandas sociales sobre estos temas. En este contexto, las estrategias áulicas que ofrecen los/las docentes están atravesadas por la sensibilidad sobre cómo se construye el conocimiento, la forma de vinculación afectiva con esos saberes compartidos y el sentido emancipador de estos.

En el caso de la UNC, la apuesta central a nivel curricular ha sido incluir el Doctorado de Estudios de Género con la pretensión de favorecer a la legitimación de los estudios de género y los estudios feministas de posgrado, así como la creación de espacios institucionales feministas (como el ya mencionado FemGes, por ejemplo). No obstante los esfuerzos mencionados, muchas de las estrategias que permiten instalar el feminismo en las aulas tienen que ver con prácticas que se realizan al margen del sistema de educación superior, como son las clases abiertas, charlas, los conversatorios, las conferencias, etc. Esto marca la agenda universitaria, pero no a los planes de estudio, que continúan sosteniendo la producción de conocimientos basados en sistemas androcéntricos.

Cuando profundizamos en relación con la población LGTBQI la representación es menor; $y$ en las prácticas áulicas quedan por fuera de lo propuesto formalmente por la UNC. En todos los casos, la adopción de la perspectiva feminista es una apuesta fuerte que discute a las modalidades académicas establecidas y legitimadas. Los feminismos vienen elaborando teorías, epistemologías y metodologías hace décadas; no obstante, los saberes patriarcales han escapado a analizar los universos posibles a través de las lentes feministas. Ello implica un reacomodamiento teórico importante, pues supone repensar trayectorias, currículos, bibliografías y producciones posibles. Pero es necesario hacerlo, debido a que tanto la historia de la ciencia como la educación se han construido al margen de las mujeres y de las sexualidades disidentes. Recuperar las narrativas, permitir que las voces de las mujeres y disidencias se escuchen, visibilizar acciones, palabras y recorridos, nos permitirá enfocar la educación y desafiar el statu quo vigente.

\section{Continuidad de diálogos}

En el marco de una educación que ha sido creada, ejecutada y diagramada por varones, las mujeres y sexualidades no hegemónicas debemos poder seguir construyendo espacios de reflexión, pensamiento y acción, disputando saberes y poderes en el marco institucional. Diana Maffía (2007) sostiene que es necesario hacer una "subversión semiótica" (p.3) para poder lograr que las mujeres pasemos, de ser objetos, a ser sujetos de enunciación. Debemos poder decir, expresar y reflexionar. Los datos nos muestran que las mujeres estudian más, califican mejor, tienen rendimiento superior; no obstante, seguimos reproduciendo prácticas patriarcales, sesgadas y eurocentradas. Además, el mejor rendimiento académico de las mujeres no repercute en las fuentes laborales porque la feminización de la pobreza sigue siendo un fenómeno expansivo en todo el mundo. Nuestros saberes están colonizados, sexualizados y racializados; nuestras universidades también. 
Las universidades, como espacios legitimadores del saber, deberían considerar a las epistemologías feministas y a las construcciones de saberes no hegemónicos. Irene Martínez (2016) sostiene que "la educación, como la cultura o la socialización, no son elementos neutrales, ya que, o bien pueden ser responsables de normalizar y reproducir sociedades desiguales, o bien pueden servir como herramientas de transformación para ciudadanías en igualdad" (4). Es importante reflexionar acerca de esto y formular la pregunta sobre qué clase de ciudadanías y de Estados construimos si la educación no representa a las mujeres y diversidades sexuales.

¿Hemos podido avanzar en el modo de inclusión de temáticas de género, feminismos y diversidad sexual en nuestras instituciones? Pensamos que la respuesta es afirmativa, y que tanto en la UNSJ como en la UNC han podido verse notables progresos en relación con la inclusión de temáticas con perspectivas de género en las aulas. La academia aún continúa revestida de androcentrismos y sexismos sin acompañar fehacientemente los procesos de cambios que se necesitan. Estas desigualdades, que aún persisten, se manifiestan en otros fenómenos dentro de las universidades, como la feminización de la responsabilidad, Ios efectos tijeras ${ }^{4}$ en la ciencia y los techos de cristal. ${ }^{5}$ Más allá de que hayamos conseguido la posibilidad de estudiar, de acceder a casas de estudios superiores y de participar en el mercado formal de trabajo, las mujeres seguimos en desventaja respecto a los varones.

Para continuar las líneas de diálogo entre nuestras universidades, podemos destacar algunas fortalezas. A partir de este trabajo (y otros que siguen la misma línea), se ha abierto la posibilidad de estrechar los vínculos entre equipos de investigación de ambas universidades que comparten intereses comunes en torno a la inclusión de contenidos con perspectiva de género y feminista, tanto en las aulas como en diversas estrategias de sensibilización en la comunidad universitaria. Otra línea de diálogo es considerar que, a pesar de las diferencias en la historia y en la cantidad de personas que conforman la comunidad académica en ambas instituciones, la presencia del patriarcado, como forma de organizar la educación superior, reproduce las mismas estrategias de exclusión hacia mujeres y disidencias en dichos ámbitos.

Asimismo, ambas universidades están dando pasos considerables en la implementación de políticas institucionales que contemplan perspectivas de género y que promueven la equidad, tales como el Observatorio y el Plan de acción frente a la violencia de género, la formación de equipos de investigación y extensión orientados hacia estas temáticas, y la sensibilización general de la comunidad universitaria en torno al género.

Por último, en estas investigaciones en diálogo hemos podido compartir que la UNC, a partir de la creación del Doctorado en Género, ha favorecido el desarrollo de líneas de investigación y acción que apoyan la inclusión de perspectivas feministas. ${ }^{6} \mathrm{Si}$ bien aún esto no se refleja en todos los programas de estudio, sí se visibiliza en las estrategias que las investigadoras/ docentes proponen. Por otra parte, la UNSJ cuenta con equipos de investigación en género ${ }^{7}$ que producen discusiones que mayormente involucran perspectivas feministas. Estos grupos, presentes en ambas instituciones, han promovido los cambios que actualmente se visibilizan en las comunidades universitarias.

Estas cuestiones nos permiten vislumbrar las posibilidades que están generando las resistencias feministas que surgen y se mantienen a lo largo de años en el marco de la Universidad.

4 Diana Maffía sostiene que el "efecto tijera" es un fenómeno estadístico que se da en algunas profesiones y trabajos. Sucede particularmente en la ciencia: "La mitad de las mujeres que entran se van abruptamente hacia abajo, y la mitad de los varones, se van abruptamente hacia arriba", sostiene Maffía.

5 El "techo de cristal" es una barrera que se les impone a las mujeres y no les permite acceder a cargos superiores.

6 En el FemGes, por ejemplo, se radican diversos proyectos de investigación con perspectiva feminista. Entre ellos están: "Violencia de género: apropiación subjetiva de derechos y reconocimiento social", dirigido por Dra. Maite Rodigou Nocetti; "Representaciones de masculinidad y femineidad: resistencias y resignificaciones", dirigido por la Dra. Cecilia Luque; "Violencia de género, representaciones y prácticas: Hacia la construcción de un marco interpretativo para analizar la violencia de género", dirigido por Dra. Alicia Soldevila; "Vulnerabilidad, desposesión y violencia normativa: El 'giro ético' de Judith Butler", dirigido por Dr. Eduardo Mattio; "Fuentes de información para el estudio de las mujeres. El archivo personal de Malvina Rosa Quiroga. Parte II", dirigido por Dra. Jaqueline Vassallo; "Género y sexualidad en la sociabilidad escolar. Un estudio de casos en escuelas medias de Córdoba", dirigido por Dra. Marina Tomasini; "Haciendo Cuerpos II. Gestión de Vidas", dirigido por Dra. Liliana Pereyra; entre otras investigaciones en curso.

7 Equipo dirigido por Laura Ávila: "Estudio sobre el debate e instrumentación de los Derechos Sexuales, Reproductivos y No Reproductivos en la provincia de San Juan (2003-2018)"- FACSO-UNSJ; también trabajo de Ana Fanchín y Patricia Sánchez: "Mujeres en la Universidad Nacional de San Juan. Presencia y protagonismo (2017) FFHA-UNSJ. 
Así como sucede en la sociedad misma, el patriarcado como estructura genera múltiples desigualdades; y la estructura universitaria no es ajena a eso. Pero, así como también pasa en la sociedad, las históricas resistencias feministas han generado cambios y acciones positivas en pos de la equidad de género que nos permiten pensar en un espacio más democrático futuro.

\section{Referencias}

Anzaldúa, G. (1998). "Este puente, mi espalda: voces de mujeres tercermundistas".

Bárcena Orbe, F., Larrosa Bondía, J. y Mèlich Sangrá, J. (2006). Pensar la educación desde la experiencia. Revista Portuguesa de Pedagogía, 233-259.

Barrancos, D. (2002). Inclusión/exclusión. Historia con mujeres. Buenos Aires, Argentina: Fondo de Cultura Económica.

Bartra, E. (septiembre-diciembre, 2018). El feminismo en las universidades. Momento: Diálogos em Educação, 28(3), 337-349.

Benavídez, A., Barboza, F., Diez, V. G., Estévez, M. F., Galoviche, V., Guerra, M.,... Pastrán, M. G. (octubre, 2018). Liderazgo y género en la educación superior: Desigualdades entre académicas y académicos en gestión. Revista Ciencias Sociales, 1(40), 67-78.

Benavídez, A., Diez, V. G., Galoviche, V., Mavrich, P. G., Guerra, M., Barboza-Pirán, F.,... Bazán, G. (diciembre, 2018). Presencia de contenidos de género en carreras de grado: El caso de la Universidad Nacional de San Juan. Entorno, (66), 102-112.

Blanco, R. (febrero, 2018). Del fulgor al desencanto. Desafíos para el feminismo académico en la fugaz experiencia de la Carrera de Estudios de la Mujer (Universidad de Buenos Aires). Revista Interdisciplinaria de Estudios de Género de El Colegio de México, 4, 01-31. doi: doi. org/10.24201/eg.v4i0.159

Bonavitta, P., Becerra, J. C., de Garay Hernández, J., Johnson, C., Wigdor, G. B., Gastiazoro, M. E.,... Sarmiento, L. (diciembre, 2018). El género en la academia: Los planes de estudio de la Universidad Nacional de Córdoba. Entorno, (66), 223-236. doi: doi.org/10.5377/entorno. v0i66.6742
Bonder, G. (1998). Los estudios de la mujer en Argentina: Reflexiones sobre la institucionalización y el cambio social. En G. Border (Ed.), Estudios de la mujer en América Latina (pp. 17-36). Washington, D. C., Estados Unidos de América: CIDI-OEA.

Buquet Corleto, A. G. (2011). Transversalización de la perspectiva de género en la educación superior: Problemas conceptuales y prácticos. Perfiles Educativos, 33, 211-225.

Espinosa, Y., Gómez, D., Lugones, M. y Ochoa, K. (2013). Reflexiones pedagógicas en torno al feminismo descolonial: Una conversa en cuatro voces. En C. Walsh (Ed.), Pedagogías decoloniales: Prácticas insurgentes de resistir, (re)existir y (re)vivir (pp. 403-441). Quito, Ecuador: Abya-Yala.

Freire, P. (1990). La naturaleza política de la educación: Cultura, poder y liberación. Barcelona, España: Ediciones Paidós.

Gargallo, F. (diciembre, 2008). El feminismo y la educación en y para nuestra América. Revista Venezolana de Estudios de la Mujer, 13(31), 17-26.

Irayigra, A. (2012). Pensando los feminismos en Bolivia. La Paz, Bolivia: Creativa.

Kirkwood, J. y Aguirre, S. M. (1987). Feminarios. Santiago de Chile, Chile: Documenta.

Kochen, S., Franchi, A. M., Maffia, D. y Atrio, J. (2001). La situación de las mujeres en el sector científicotecnológico de América Latina. Principales indicadores de género. En E. Pérez Sedeño (Ed.), Las mujeres en el sistema de ciencia y tecnología (pp. 19-39). Madrid, España: OEl.

Korol, C. (2007). Hacia una pedagogía feminista: Géneros y educación popular. Buenos Aires, Argentina: El Colectivo, América Libre.

Korol, C. (abril, 2017). Cuerpos y territorios. Las mujeres en la defensa de la vida diversa y de la libertad. Conferencia presentada en las memorias de las III jornadas nacionales de ecología política, San Juan, AR.

Labarca, A. (1947). Feminismo contemporáneo. Santiago de Chile, Chile: Zig-Zag.

Lagarde, M. (2000). Claves feministas para liderazgos entrañables. Managua, Nicaragua: Puntos de Encuentro. 
Lamas, M. (2013). El género: La construcción cultural de la diferencia sexual. México, D. F., México: Miguel Ángel Porrúa.

Lamas, M. (noviembre, 1986). La antropología feminista y la categoría género. Nueva Antropología, 8(30), 173-198.

Maffía, D. (junio, 2007). Epistemología feminista: La subversión semiótica de las mujeres en la ciencia. Revista Venezolana de Estudios de la Mujer, 12(28), 63-98.

Martínez Martín, I. (enero-junio, 2016). Construcción de una pedagogía feminista para una ciudadanía transformadora y contra-hegemónica. Foro de Educación, 14(20), 129151. doi: doi.org/10.14516/fde.2016.014.020.008

Mendoza, B. (2014). Ensayos de crítica feminista en nuestra América. México, D. F., México: Herder.

Moraga, C. y Castillo, A. (1998). Este puente, mi espalda: voces de mujeres tercermundistas en los Estados Unidos. San Francisco, Estados Unidos de América: Ism Press.

Morgade, G. (1997). Mujeres en la educación, género y docencia en Argentina, (1870-1930). Buenos Aires, Argentina: Miño y Dávila.

Morgade, G. (2011). Toda educación es sexual: Hacia una educación sexuada justa. Buenos Aires, Argentina: La crujía.

Morgade, G. (mayo, 2018). Las universidades públicas como territorio del patriarcado. Política Universitaria, 1(5), 32-43.
Organización Internacional del Trabajo. (2016). Las mujeres en el trabajo [versión de Adobe Acrobat Reader]. Recuperado de https://www.ilo.org/wcmsp5/groups/ public/---dgreports/---dcomm/---publ/documents/ publication/wcms_483214.pdf

Palermo, Z. (2007). Desafíos éticos para la Universidad Latinoamericana del futuro. En H. E. Biagini y A. A. Roig (Comps.), América Latina hacia su segunda independencia: Memoria y autoafirmación (pp. 297308). Buenos Aires, Argentina: Aguilar, Altea, Taurus, Alfaguara.

Pérez Sedeño, E. (2001). Las mujeres en el sistema de ciencia y tecnología: Estudios de casos. Madrid, España: OEI.

Putnam, R. y Borko, H. (2000). El aprendizaje del profesor: Implicaciones de las nuevas perspectivas de la cognición. En B. J. Biddle, T. L. Good y I. F. Goodson (Coords.), La enseñanza y los profesores (pp. 219-308). Barcelona, España: Paidós Ibérica.

Valcárcel, A. (2008). Feminismo en el mundo global. Madrid, España: Cátedra.

Viveros Vigoya, M. (2009). La sexualización de la raza y la racialización de la sexualidad en el contexto latinoamericano actual. Revista Latinoamericana de Estudios Familiares, 1(4), 63-81.

Walsh, C. (2009). Interculturalidad crítica y pedagogía decolonial: Apuestas (des) de el in-surgir, re-existir y revivir. Revista (Entre Palabras), 3, 1-29. 